\title{
STRUCTURE-FROM-MOTION PROCESSING OF AERIAL ARCHIVE PHOTOGRAPHS: SENSITIVITY ANALYSES PAVE THE WAY FOR QUANTIFYING GEOMORPHOLOGICAL CHANGES SINCE 1978 IN LA RÉUNION ISLAND
}

\author{
C. Rault ${ }^{1}$, T.J.B. Dewez ${ }^{1 *}$, B. Aunay ${ }^{1}$ \\ ${ }^{1}$ BRGM -French Geological Survey, 45060 Orléans-la-Source, France - (c.rault, t.dewez, b.aunay)@brgm.fr
}

KEY WORDS: SFM, archive aerial photograph, camera calibration, landslides, cyclone, La Réunion Island

\begin{abstract}
:
Landscapes have been photographed dozens of times at scales ca. 1/25,000 and better since World War II. Scans are distributed freely online (e.g. remonterletemps.ign.fr). In parallel, Structure-from-Motion (SFM) software made photogrammetric processing easy to non-specialists. Yet puzzling questions crop up to use both: (i) Can raw scans be used as is? (ii) Can Ground Control Points (GCP) and checkpoints be safely collected from a web portal? (iii) How many parameters are sufficient for camera interior orientation? (iv) Are single flight camera networks sufficient to constrain camera models compared to multiple flights? (v) Are photogrammetric Digital Surface Models (DSM) fit for quantifying landslide activity? Processing of scanned black-and-white 1/27,000 photographs from IGN flown in May 1978 over Cirque de Salazie in La Réunion Island answer these questions. We find that raw scanned photographs need translation, rotation and cropping to match the camera reference frame. GCP and Check point coordinates collected on geoportail.gouv.fr with assumed accuracy of $10 \mathrm{~m}$, achieved ca. $7 \mathrm{~m}$ accurate SFM registration. The optimal camera model uses only 4 parameters: f, cx, cy and $\mathrm{K}_{1}$. Compared to a 2015 lidar Digital Terrain Model (DTM), the $0.66 \mathrm{~m} /$ pixel DSM of 1978 has a median deviation of $-1.39 \mathrm{~m} \pm 3.34 \mathrm{~m}$ (Median Absolute Deviation) which is comparable to GCP quality. Elevation difference more importantly reveals, for the first time, the 37 years and 13 cyclones cumulated landslides pattern on Cirque de Salazie. Photographic archives hold decades-long 3D history. SFM is a game changer for landslide risk mitigation planning.
\end{abstract}

\section{INTRODUCTION}

Structure-from-Motion (SFM) is a method widely applied in geoscience to produce topographic data of a landscape from digital photos (Eltner et al., 2016). Such data is useful to extract information from the landform and monitor its evolution over time (e.g. Cook, 2017; Dewez et al., 2016; James and Robson, 2012; Johnson et al., 2014; Smith and Vericat, 2015).

Systematic aerial photogrammetric surveys image the earth surface since the middle of the 20th century, every few years. Freely distributed online in many countries, geomorphologists are longing to exploit this archive to build historical digital surface models and accurate ortho-photographs to retrieve quantitative landscape evolution metrics. Recently, it has been demonstrated that Structure-from-Motion software could leverage this information to quantify landscape changes (Bakker and Lane, 2016; Gomez et al., 2015; Micheletti et al., 2015; Riquelme et al., 2019).

Structure-from-Motion originated in computer vision where 3D quantitative robustness was achieved through redundant oblique photographs (e.g. Ma et al., 2004). This SFM paradigm, however, does not fit archive aerial surveys, with their weak geometry, all parallel nadir-looking axes with minimal stereoscopy (60\% overlap along-track and 30\% across-track). The reason for this weak geometry is that large format photogrammetric film was expensive to purchase and develop, and that stereo interpretation was done by human operators one pair of photos at a time. While valuable in principle, archives of aerial photographs are, a priori, ill-suited for SFM processing.

As for the models derived from numerical photographs from the ground and/or aerials vehicles, some limitations induced errors in the final reconstruction models as distortions, missing data, and blurry areas (e.g. Fonstad et al., 2013; James and Robson, 2014b).

The main limitations evoked in the literature against historical photos include:

The quality of the scanned original photographs: scratches, dust and stains on the original negatives, blurred due to defect in film flatness on the focal plane and ill-exposed pictures are sources of errors in the reconstruction (Gomez et al., 2015).

- Camera resolution and/or digitization resolution: a low resolution limits small ground feature recognition and cross-photograph matching. This may generate false fixes that create spurious elevation artefacts (Gomez et al., 2015; Riquelme et al., 2019). For reference, Gomez et al. (2015) used photos with pixel resolution of $63.5 \mu \mathrm{m}-127 \mu \mathrm{m}$ (400 to 200 dpi, i.e. theoretical GSD, ground sampling distance, between $0.63 \mathrm{~m}$ and $3.81 \mathrm{~m}$ ), while Riquelme et al. (2019) scanned photos at $42.3 \mu \mathrm{m}$ (600 dpi, theoretical GSD: $1.05 \mathrm{~m})$;

- Picture overlaps: The larger the overlap, the denser the point cloud generated and the more detailed the topographic model should be if the generated points are accurate (Westoby et al., 2012). From theoretical simulations, James and Robson (2014b) showed that pictures taken from UAVs with parallel viewing direction may contain impossible-to-resolve camera modelling residuals except by adding costly additional ground control points or introducing oblique photographs;

- Ground Control Points (GCP). A minimum of three referenced points is required to scale and transform a photogrammetric model into a geodetic system (James and Robson, 2012). The quality of the reconstruction

\footnotetext{
${ }^{*}$ Corresponding author
} 
increases with the number and the quality of ground control points (Barrand et al., 2009; James and Robson, 2012). Location errors may creep in if the technology used to collect the GCP is inaccurate. This can be described by the superiority ratio (Eltner et al., 2016). Further, a user could flag a GCP at the wrong location in the image (Riquelme et al., 2019), leading to gross orientation errors. Gomez et al., 2015 recommended a minimum of 10 to 15 GPCs spatially well-distributed to mitigate errors in the model build from historical images.

So here we tackle the issue on a particular data set coming from La Réunion Island, using ca. 1/27,000 aerial photographs shot in May 1978 by IGN, the French Geographic Survey. This date precedes the dramatic Hyacinthe cyclone event of January 1980. The cyclone's chaotic course had it stay nearly two weeks in the vicinity of the island, causing 25 casualties. Heavy rainfalls, $6083 \mathrm{~mm}$ rainfall in 15 days, triggered deadly landslides and the sudden, abnormally fast, 720-m-long headward erosion of Ravine de l'Eglise on Grand-Ilet landslide (Pinchinot, 1984) (Figure 1). Archive photographs flown two years before the cyclone document the forever-gone landscape, offering insight into the conditions preceding the dramatic event. The tropical island of La Réunion is characterized by elevations reaching $3069 \mathrm{~m}$ above sea level encroached by clouds in a somewhat unpredictable fashion. This adverse meteorological phenomenon opportunistically turns into a SFM advantage since several aerial survey flights are sometimes necessary to patch together a cloudless coverage for the whole of the island. We argue that these multi-date surveys improve redundancy for SFM-optimal conditions. Using these flight properties, we investigate selfcalibrating bundle adjustment behaves in SFM software Agisoft Metashape v1.5.2, which is used by a large community.

In this contribution, we ask and answer a series of practical questions: (i) Can raw photo scans be readily used without preprocessing? (ii) Can GCP be collected effectively on a webmapping portal? (iii) How many parameters are sufficient to parametrize the camera interior orientation? (iv) Is minimal stereoscopic coverage (single flight) sufficient to constrain the camera model compared to more redundant multiple flight configuration? (v) Are photogrammetric Digital Surface Models fit for quantifying landsliding?

\section{STUDY AREA AND MATERIALS}

\subsection{Study area}

La Réunion is a volcanic island located in the Indian Ocean ca. $700 \mathrm{~km}$ East from Madagascar. The highest point, Piton des Neiges, culminates at $3069 \mathrm{~m}$ above sea level, $24 \mathrm{~km}$ from the coast. The slope gradient averages at $12.5 \%$. Three large depressions called "cirques" occupy the centre of the island and radiate from the summit. The Cirque de Salazie is the cirque located North of Piton des Neiges. Its surface area is about $100 \mathrm{~km}^{2}$. The cirque is skirted by more than 500-meters-high cliffs called "remparts". The central part of the cirque is affected by multi-million cubic meters landslides (e.g. Grand-Ilet and Hell-Bourg landslides) and multiple smaller landslides and mass wasting sites. This physiographic configuration introduces two challenges for historical photogrammetry: (i) stable areas occupy a very limited portion of the landscape, which necessitates prior knowledge for collecting robust GCP coordinates today; (ii) the rempart elevation above the cirque floor creates deep shadows and large image parallax, often larger than can be accommodated by the $60 \%$ stereoscopic overlap of the survey. This leaves unresolved 3D data gaps on the steep rocky slopes.

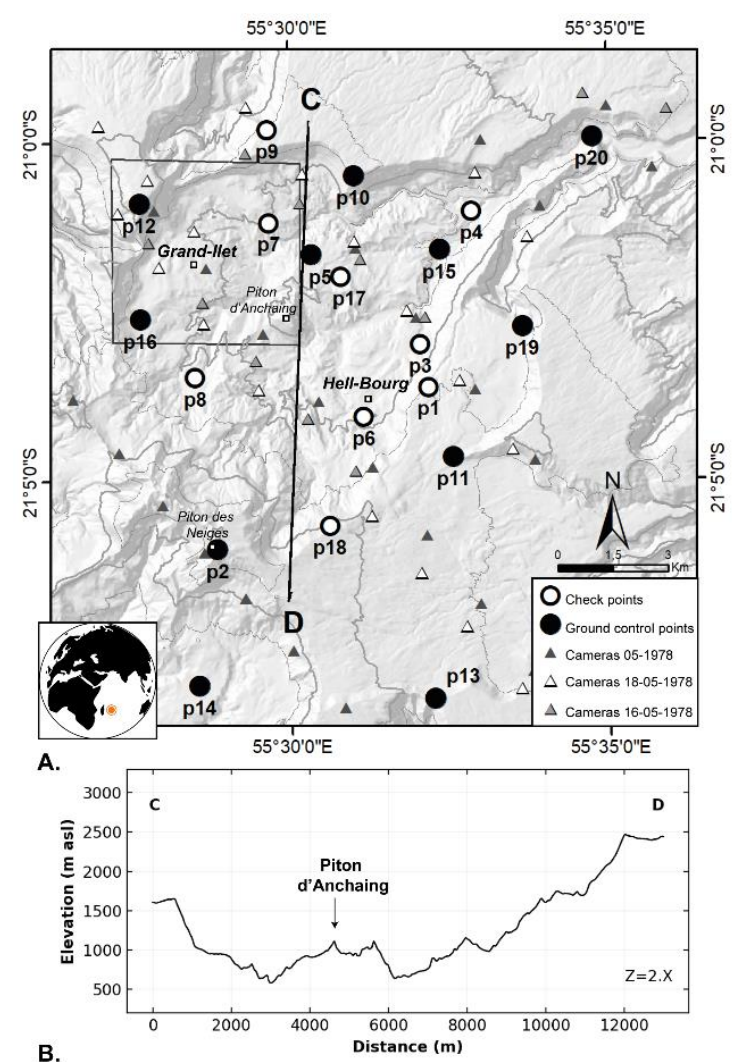

Figure 1. Cirque de Salazie camera network of 1978. A set of 11 GCP bracket the area of interest, as well as 9 checkpoints verify model adjustment. Small triangles figure the 60 camera locations used in this work. Flight lines are oriented NW to SE. The black line C-D mark the ends of the topographic profile presented in B. The black rectangle delimits the extent of Figure 9 and Figure 10.

Between p5 and p17, indicates the profile of Figure 7

\subsection{Data used}

Between 1949 and 2011, 20 vertical aerial photo campaigns covered Cirque de Salazie. That is more than one in every 4 years. Digital files are distributed free-of-charge on IGN's portal http://remonterletemps.ign.fr as JPEG2000 files. We focus our analysis on the 6 aerial surveys of May 1978 (Table 1), as they predate the occurrence of cyclone Hyacinthe of January 1980.

Calibration certificate of the cameras are not distributed online alongside the freely downloadable photos. Processing thus has relied on self-calibrating bundle adjustments to determine camera parameters. By contrast, Agisoft Metashape (and earlier Agisoft Photoscan versions) implements Brown's (1971) camera model where radial distortion is determined with coefficients $K_{(1,2,3)}$ of an even-order polynomial (i.e. $r^{2}, r^{4}, r^{6}$ ) with respect to a single, but different, principal point. Eccentricity values or $K$ and $k$ should absolutely not be confused, they reflect different realities. So obtaining calibration certificates would only instruct us about the fiducial mark positions with respect to the camera reference frame, other information would have been irrelevant for Brown's camera model. 


\begin{tabular}{|c|c|c|c|c|c|}
\hline Date & $\begin{array}{c}\mathrm{Nb} \\
\text { photos }\end{array}$ & $\begin{array}{c}\mathrm{Nb} \\
\text { Bands }\end{array}$ & $\bar{h}(\mathrm{~m})$ & $\mathrm{b} / \mathrm{h}$ & $\begin{array}{c}\text { GSD } \\
(\mathrm{mm})\end{array}$ \\
\hline 11-May-1978 & 2 & 1 & 4080 & 0.51 & 533 \\
\hline 13-May-1978 & 9 & 1 & 3915 & 0.51 & 511 \\
\hline 15-May-1978 & 9 & 2 & 4345 & 0.53 & 567 \\
\hline 16-May-1978 & 12 & 3 & 3975 & 0.50 & 519 \\
\hline 18-May-1978 & 21 & 4 & 4305 & 0.53 & 562 \\
\hline 22-May-1978 & 7 & 2 & 4570 & 0.55 & 596 \\
\hline
\end{tabular}

Table 1. Details of 1978 archive photographs available on http://remonterletemps.ign.fr on Cirque de Salazie, La Réunion, used in this study. All photos were shot with an unknown camera equipped with a UAgI $153.23 \mathrm{~mm}$ lens (serial number 6023). $\bar{h}$ is the height above ground average of the camera centers. $b / h$ is the average overlapping of the camera and the ground sampling distance (GSD) $(0.02 . \bar{h} / \mathrm{f} \mathrm{mm})$. Taking the 38 pictures with multiview stereo b/h equals to 0.49 and GSD $557 \mathrm{~mm}$.

The 9 inches argentic analog photographs were scanned by IGN with a probable pixel pitch of $20 \mu \mathrm{m}$ with an undocumented device. We guess the pitch from the image distance between fiducial marks: ca. 10,000 pixels for $20 \mathrm{~cm}$ measured with a ruler on a printed photo.

\section{PREPARATION OF THE AERIAL PHOTOGRAPHS}

Previous work using archive photographs with SFM software (Gomez et al., 2015; Riquelme et al., 2019) were somewhat elusive regarding their image preparation. Scanned file comparison shows that film placement on the scanner's window was not strictly reproduced. Cropped larger than film extent, the images cannot be readily mapped to a unique camera reference system. Although Agisoft Metashape can handle fiducial mark objects, these could not be used in this project because the software expects calibrated mark coordinate values. Without camera calibration certificate, fiducial positions are unknown. This is why we wrote a Python code to perform the inner orientation task.
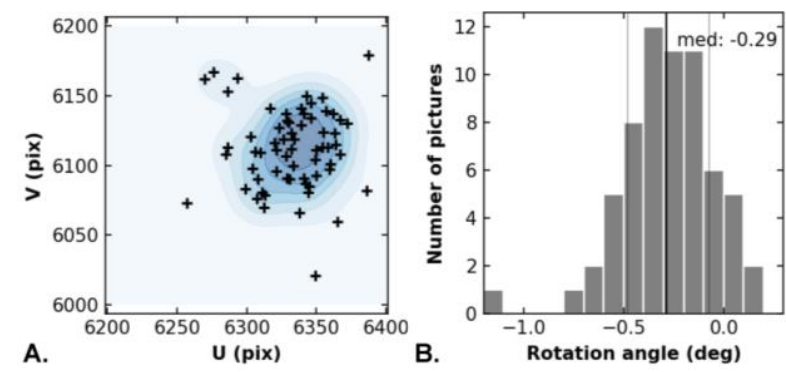

Figure 2. A. Position of the camera fiducial diagonals intersection in the original image reference frame. This central point varies in a domain of 150 x 150 pixels. B. Rotation angle applied to scanned images to align image coordinates to fiducial marks. The median (-

$\left.0.29^{\circ}\right)$ and the $17 \%$ and $18 \%$ quantiles $(-0.48$ and -0.075$)$ are

represented by the black and grey vertical lines. These show that translate, rotate and crop operations are necessary to align images to the camera reference frame.

Our code reads JPEG2000 images, automatically detects fiducial marks with one pixel accuracy, with pattern matching correlation using Lucas-Kanade method, rotates the image coordinates so that XY camera axes align to image lines/columns and crops the images to the same origin (upper left corner fiducial). Cropped images, removing the black film edge, are output as lossless TIF files. In this way, the camera principal point should always occur in a unique image location corresponding to the true photo center.

Figure 2 shows that individual photo image coordinates are indeed very variable from one scan to the next: individual photo coordinate origin on the scanned image vary by up to 150 pixels $(3 \mathrm{~mm})$, and rotation spans $1^{\circ}$ of rotation. Assigning all images to being captured with the same camera, Metashape expects that all images reflect a unique, static, image coordinate system. If Metashape had managed to calibrate such poor camera situation at all, calibration results would still be grossly erroneous. Our Python code automates this inner orientation pre-processing. Its validity was checked manually with 40 fiducials. $88 \%$ of estimates match within 1 pixel, the precision limit vouch for our fiducial manual identification.

\section{SFM-BASED CAMERA CALIBRATION}

Aerial photogrammetric surveys were historically designed to shoot minimally overlapping photos with parallel and nadirlooking axes images (60\% along-track overlap, 30\% across-track side lap, e.g. Kraus, 1993). 3D reconstruction by SFM, by contrast, is optimal if the photo sets contain oblique photographs and large coverage redundancy (e.g. James and Robson, 2014b; Wenzel et al., 2013). Historical photograph networks are considered weak photogrammetric network (James et al., 2017). Nevertheless, air photo archives contain such a wealth of landscape evolution information and SFM softwares integrate much improved dense matching algorithm nowadays, that investigating their combined use is worth being framed again.

We therefore explore to what limits SFM-derived Digital Surface Models (DSM) are appropriate tools for geomorphologic application and change detection. While SFM reconstruction and change detection has already been discussed previously by e.g. Gomez et al. (2015) or Riquelme et al., (2019), image redundancy, or lack thereof, on self-calibrating bundle adjustment still requires exploring (Aguilar et al., 2013).

The question tackled is: Is weak geometry inappropriate for quantifying geomorphic changes over several decades?

To initiate the SFM exercise, a collection of 20 reference points were digitized on IGN's cartographic portal geoportail.gouv.fr. We resorted to this option because this is an increasing temptation to use all available information online. This approach is relevant for many geomorphologists out there, who generate data prior to conducting fieldwork. In the absence of formal statement by IGN about the quality of coordinates displayed online, we reasonably assume point coordinates accuracy of $10 \mathrm{~m}$ in $\mathrm{XYZ}$ in the bundle adjustment.

The reference points were selected in flat, otherwise suspected stable areas, and in positions bracketing the 3D domain of interest (Figure 1) (Kraus, 1993): i.e. at least pinning all four corners of the area and constraining an elevation span between $266 \mathrm{~m}$ and $3066 \mathrm{~m}$ above sea level. Coordinates were encoded in RGR92 UTM40S (EPSG code: 2975). The set of reference points was split between 11 Ground Control Points (GCP), and 9 checkpoints to validate the accuracy of the exterior orientation. After the initial orientation process ("Align" operation in Metashape), which uses GCPS coordinates in the bloc bundle adjustment, reference points had precision residuals of 0.72-0.90 pixels (Table 2). This precision remained stable whether the "Medium" or "High" accuracy setting was selected (Table 2). 


\begin{tabular}{|c|c|c|c|c|}
\hline & \multicolumn{2}{|c|}{$\begin{array}{c}\text { Align } \\
\text { medium accuracy }\end{array}$} & \multicolumn{2}{|c|}{$\begin{array}{c}\text { Align } \\
\text { high } \text { accuracy }\end{array}$} \\
\hline & $\begin{array}{c}11 \\
\text { GCP }\end{array}$ & 9 Check & $11 \mathrm{GCP}$ & 9 Check \\
\hline $\begin{array}{ll}\text { Align } & \text { (no } \\
\text { optimize) }\end{array}$ & $\begin{array}{l}7.301 \mathrm{~m} \\
0.906 \mathrm{pix}\end{array}$ & $\begin{array}{c}6.76 \mathrm{~m} \\
0.801 \text { pix }\end{array}$ & $\begin{array}{c}7.56 \mathrm{~m} \\
0.720 \text { pix }\end{array}$ & $\begin{array}{c}5.79 \mathrm{~m} \\
0.779 \text { pix }\end{array}$ \\
\hline $\begin{array}{l}3 \text { params (f, } \\
\text { cx, cy) }\end{array}$ & $\begin{array}{c}7.04 \mathrm{~m} \\
0.196 \text { pix } \\
\end{array}$ & $\begin{array}{c}6.86 \mathrm{~m} \\
0.203 \text { pix } \\
\end{array}$ & $\begin{array}{c}7.08 \mathrm{~m} \\
0.324 \text { pix } \\
\end{array}$ & $\begin{array}{c}5.48 \mathrm{~m} \\
0.328 \text { pix } \\
\end{array}$ \\
\hline $\begin{array}{l}4 \text { params } \\
\left(f, c x, c y, K_{1}\right)\end{array}$ & $\begin{array}{r}7.18 \mathrm{~m} \\
0.196 \mathrm{pix} \\
\end{array}$ & $\begin{array}{c}7.04 \mathrm{~m} \\
0.203 \text { pix }\end{array}$ & $\begin{array}{c}7.03 \mathrm{~m} \\
0.324 \text { pix }\end{array}$ & $\begin{array}{c}5.45 \mathrm{~m} \\
0.328 \mathrm{pix} \\
\end{array}$ \\
\hline $\begin{array}{l}8 \text { params (f, } \\
\text { cx, cy, } \mathrm{K}_{1}, \mathrm{~K}_{2}, \\
\left.\mathrm{~K}_{3}, \mathrm{P}_{1}, \mathrm{P}_{2}\right)\end{array}$ & $\begin{array}{r}7.33 \mathrm{~m} \\
0.191 \text { pix }\end{array}$ & $\begin{array}{c}7.26 \mathrm{~m} \\
0.198 \text { pix }\end{array}$ & $\begin{array}{c}6.98 \mathrm{~m} \\
0.324 \text { pix }\end{array}$ & $\begin{array}{r}5.46 \mathrm{~m} \\
0.319 \mathrm{pix}\end{array}$ \\
\hline
\end{tabular}

Table 2. Reference point residuals obtained when performing alignment of the 60 images with two accuracy modalities (Medium vs High), and computing self-calibrating bundle adjustment (Align result or Optimize for 3, 4 or 8 parameters). The retained solution is in bold. Camera models with more than 4 parameters do not improve point residuals significantly because model precision is mostly sensitive to camera network. Cameras are satisfactorily modelled with principal distance (f), and eccentricity (cx, cy) and a radial distortion parameter $\left(\mathrm{K}_{1}\right)$. Adding other parameters does not improve results.

From the results of Table 2, we draw several conclusions. First, using Medium or High accuracy in the Align procedure does not change drastically adjustment quality. This finding may not have a generic character, but with the photos of 1978 in black and white, scanned at a $20 \mu \mathrm{m}$ pitch, Medium accuracy (i.e. matching tie points identified on 4-times-downsampled images) is as precise as with High accuracy (i.e. tie points matched on full resolution images) for alignment.

Second, residuals for GCP and checkpoints are closely tied to each other. This shows that interior and exterior orientation provide residuals smaller than $10 \mathrm{~m}$ in object space (Table 2), the assumed reference point accuracy. The superiority ratio is smaller than one, $7 / 10=0.7$ (see Eltner et al., 2016).

Third, optimizing camera calibration, as opposed to keeping raw alignment results, improved point residuals by a factor of 2 to 3 . Point residuals dropped from $0.906-0.801$ pixels to $0.319-0.324$ pixels, depending on the parameter configuration (Table 2). Optimization of the camera parameters, step in which GCPs are also considered, brings therefore a gain in precision as expected (James and Robson, 2012; Eltner and Schneider 2015).

Fourth, camera calibration parameters are always a puzzling issue to non-specialists. James et al. (2017) insist that over parametrization should not be attempted. Correlated parameters (i.e. with correlation coefficients exceeding 0.1 ) could be dropped. This is what is observed here. A camera model optimizing only the principal distance $f$ and principal point eccentricity (cx, cy) achieves sub-pixel residuals just as well as more complex models involving radial distortion alone $\left(\mathrm{K}_{1}\right)$ or radial and tangential distortions $\left(\mathrm{K}_{1-3}\right.$ and $\left.\mathrm{P}_{1-2}\right)$. However, keeping one distortion parameter $\left(\mathrm{K}_{1}\right)$ avoid to end up with a doming effects (Eltner and Schneider, 2015).

\section{CAMERA PARAMETER SENSITIVITY TO REDUNDANT COVERAGE}

Because Metashape offers the possibility to interact programmatically through its Python API, further tests were run to assess the robustness of camera calibration parameters and the impact of redundant camera network on calibration parameters. For this, a bootstrapping method was employed. A reference "chunk" containing all 60 aligned photos with high accuracy contained a set of 63079 tie points with a multiplicity of 2.64 in average, 11 GCP and 9 checkpoints. $85 \%$ of the reference points are observed on more than four photos. A bootstrapping method reproduced one hundred times the following: a random subset of $70 \%$ of tie points was sampled and an optimize operation run for $\mathrm{f}$, cx, cy and $\mathrm{K}_{1}$. The camera network (positions and angles) was saved, along with reference point residuals, number of unique tie points effectively sampled and camera calibration parameters. This procedure was employed to test: (i) whether camera calibration parameter computed with $100 \%$ of tie points were stable, and (ii) how single date flight, thus poorer stereoscopic conditions, performed comparably to multiple date flights.

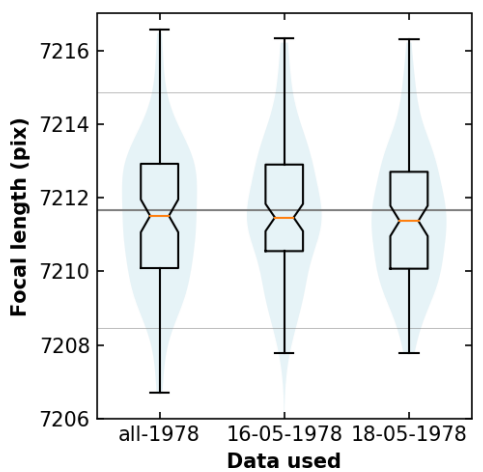

Figure 3. Violin-plots of principal distance estimated with 100 bootstraps, sampling $70 \%$ of initial tie points. The solid horizontal line represents the principal distance estimated once with $100 \%$ of tie points (67 073 points). The thin horizontal is the $1-\sigma$ error on

principal point estimate. Three situations are tested. All-1978 estimates the principal distance using all photos flown in 1978, thus more redundant than classically minimal stereoscopic conditions

(60\% overlap, 30\% side lap). 16-05-1978 estimates the focal distance only using 12 photos in 3 flight bands with $60 \%$ overlap and 30\% side-lap. 18-05-1978 estimates using 21 photos in 4 flight bands with $60 \%$ overlap and $30 \%$ side-lap.

Figure 3 presents the bootstrapping results for the principal distance. Whether one uses the entire stereoscopic set of photographs (60 photos flown in 6 flights), or a single flight (16 or 18 May 1978) (see Table 1), the computed principal distance is comparable. Bootstrapped values are slightly lower than the value assessed using $100 \%$ of tie points, but they all lie between the 1-sigma and 2-sigma limits of the reference value. They are not significantly different. We conclude from this that additional stereoscopic overlap does not alter camera calibration parameter estimates. The range of principal distance values is susceptible to change by about 10 pixels over 7212 pixels. This corresponds to a relative precision of ca. 1/1000.

Testing the principal point (Figure 5) shows that it is rather stable to within $0.1-0.15$ pixels.

Model exterior orientation accuracy could also be questioned. Bootstrapping results therefore captured model residuals for reference points (Figure 5). No remarkable influence can be noticed between error histograms. All simulations have a mode at $6.8 \mathrm{~m}$ and a span between $6.65 \mathrm{~m}$ and $7.13 \mathrm{~m}$. Given that reference points coordinates were obtained on the most recent 
orthophotos of geoportail.gouv.fr and elevation interpolated from the reference digital elevation model (actual elevation reference not documented on the website), we deemed coordinates to be correct to within $10 \mathrm{~m}$ at best. Bootstrap simulations are coherent with this assumption.

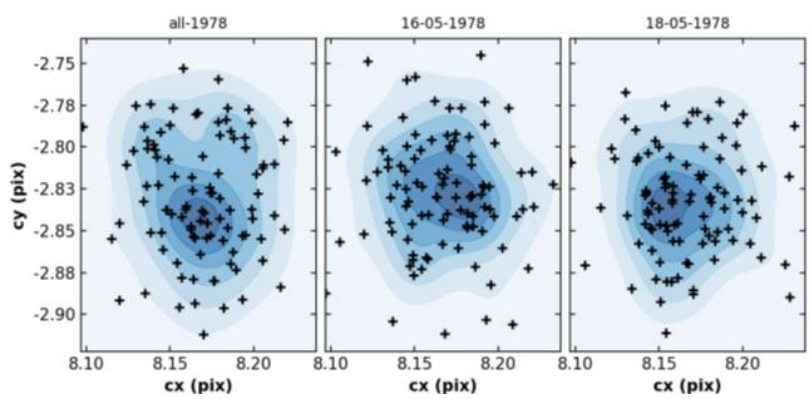

Figure 4. Eccentricity (cx, cy) parameters, relative to image centre, from 100 independent bootstrap samples of $70 \%$ of tie points using either, all photos, 16-May or 18-May flights. Variations remain small (0.15-0.2 pixel). With $100 \%$ of tie points (67 073 points) the parameters equal to $\mathrm{cx}=8.24$ and $\mathrm{cy}=-2.75$.

Having observed that the photogrammetric process was working reliably, the last step consists in checking whether digital surface models (DSM) extracted from 1978 bear some resemblance with present day elevation reference.

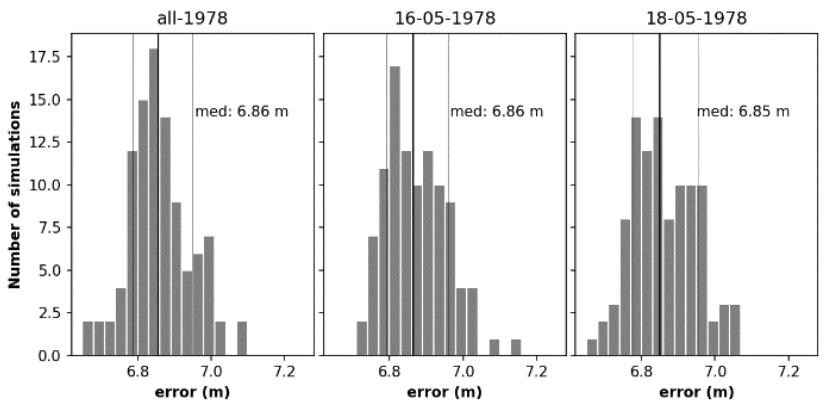

Figure 5. Residual error of GCP (in m) resulting from 100 bootstrap samples alignment and optimization. All simulations consistently have a median at $6.85-6.86 \mathrm{~m}$. This tends to show that stereoscopic conditions when using all 60 photos or only the flight

of 16 or 18 May 1978 do not alter exterior orientation in a significant fashion.

\section{DSM COMPARISON AND LANDSCAPE EVOLUTION}

The SFM pipeline was further unfolded to extract dense point clouds with UltraHigh/aggressive, filtering settings recommended by Metashape for aerial photos, all 60 photos of 1978 and a camera model with 4 parameters (f, cx, cy, $K_{1}$ ). The "DEM" (in the sense of the Metashape function) was processed at the default resolution of $0.66 \mathrm{~m} /$ pixel. But to limit noise, comparison were conducted with the DSM Geotif grid resampled at $5 \mathrm{~m}$, using bilinear interpolation in ArcGIS.

The 1978 DSM was compared to a reference lidar elevation dataset acquired for La Réunion Regional Council in 2015 (Figure 6). According to the comparison with 25 geodetic markers distributed in the Cirque, this MNT is accurate at $18 \mathrm{~cm}$ $+/-12 \mathrm{~cm}$ in elevation. This helicopter-borne lidar data set was acquired with an average point density of $67.5 \mathrm{pts} / \mathrm{m}^{2}$, with accuracy requirements at 1 -sigma of $20 \mathrm{~cm}(\mathrm{XY})$ and $10 \mathrm{~cm}(\mathrm{Z})$.
After point classification, point density dropped at $3 \mathrm{pt} / \mathrm{m}^{2}$ on average. The delivered Digital Terrain Model (DTM) raster was interpolated from ground points at $0.5 \times 0.5 \mathrm{~m}$ resolution. To compare with 1978, this DTM was also resampled at $5 \mathrm{~m}$. The Difference of DEM (DoD, a common on notion in geomorphology) is presented in Figure 6. Note that, although we could have used this quality data set for collecting accurate reference points, we left it aside to explore the limitations of fully web-based sources. Elevation model comparisons are therefore fully independent.

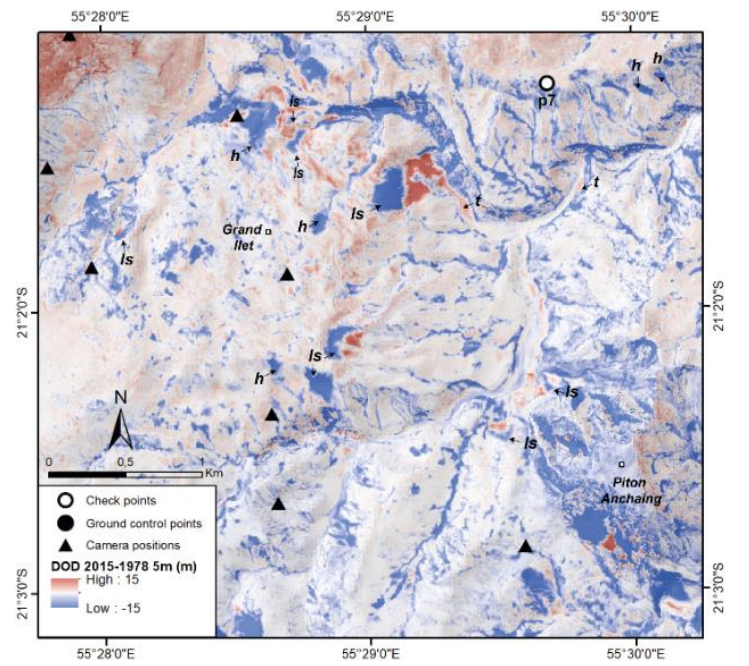

Figure $6.5 \times 5 \mathrm{~m}$ Difference of DEM (DOD) comparing a heliborne lidar DTM acquired in 2015, originally at $0.5 \times 0.5 \mathrm{~m}$ resolution, and the 1978 (all photos, 4 parameters calibration) photogrammetric coverage produced at $0.66 \mathrm{~m} /$ pixel. Deep blue (erosion) and red

zones (accumulation) reveal major geomorphic or anthropic changes effectively known around Grand Ilet sector in Cirque de Salazie. Some remarkable landforms are pointed by black arrows ls: landslide, h: headward erosion, t: river terraces. The most visible landslide (dual blue/red patch in the centre north) is the $1 \mathrm{Mm}^{3}$ Roche à Jacquot landslide triggered by cyclone Béjisa in 2014.

The pattern of elevation change between 2015 and 1978 shows spectacular pockets of negative values (Figure 6). This pattern of high landslide activity is coherent with the fast displacement rates known in the area. From DGNSS permanent and monitoring campaigns surveys benchmarks, it is known that Grand Ilet experiences $0.05 \mathrm{~m} / \mathrm{yr}-0.52 \mathrm{~m} / \mathrm{yr}$ horizontal motion on up to $15^{\circ}$ average slopes, vertical displacement of $0.01 \mathrm{~m} / \mathrm{yr}$ to $0.13 \mathrm{~m} / \mathrm{yr}$ (Belle et al., 2014). It is not surprising that Cirque de Salazie exhibits such massive changes in 37 years.

In more quantitative terms, Figure 7 shows the elevation change observed in a narrow $(20 \mathrm{~m} \times 1000 \mathrm{~m}) \mathrm{N}-\mathrm{S}$ box located in a stable area (location between reference points p5 and p17, Figure 1) illustrates that there is a fair agreement between elevations estimated by photogrammetry in 1978 and lidar in 2015. The photogrammetric data is biased by $-1.39 \mathrm{~m}$ with a median absolute deviation (descriptor advocated by Höhle and Höhle, 2009) equals to $3.34 \mathrm{~m}$ (Figure 7). Characteristic elevation difference quantiles (q17\% and q83\%, therefore bracketing $2 / 3$ of the elevation samples) are respectively $-6.92 \mathrm{~m}$ and $1.12 \mathrm{~m}$. Admittedly, elevation discrepancies are sizeable. Beyond identifying ground movements (landslides and erosion), our approach is valid to assess with a $5 \mathrm{~m}$ resolution volumes of landslides and eroded areas. This precision may seem coarse compared to today's lidar accuracy. But photogrammetry 
provides a landscape-wide topographic image for a period at which none existed so far. It is pretty amazing.

Elevation errors arise for several reasons. First, a photogrammetric surface model is compared to a lidar terrain model. Elevation on tropical vegetation is susceptible to explain a significant part of the elevation errors. Second, the ground control points used to georeference the photogrammetric dataset show an agreement to within $7 \mathrm{~m}$. Better GCPs with a proper DGNSS survey will improve georeferencing and lead to smaller probably unbiased elevation errors (Figure 7B). Yet, our procedure exploiting solely online data for an island $9,000 \mathrm{~km}$ from mainland Europe, shows it is an invaluable resource to prepare fieldwork.

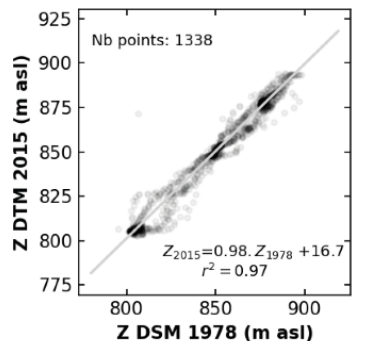

A.

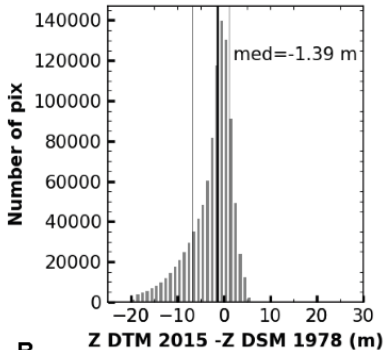

B.
Figure 8 shows the histograms of the elevation differences between the DTM 2015 and the DSM 1978 at low and high elevation. The distribution median are also biased, yet slightly less than the 1978 DSM including all photos (Figure 9 and Figure 10). Distribution characteristic quantiles (17\% and 83\%) show that low elevation 1978 DSM is more focused, though both show a quantile span exceeding slightly $10 \mathrm{~m}$. More importantly, the median error is smaller for the lower flight DSM. This is in line with the scaling of photographs. Lower flight means better imaging scale and thus better elevations.

To reveal more profound differences between coverages, we computed the difference between 1978 DSMs (All - low elevation and All - high elevation) (Figure 9 and Figure 10). The elevation difference patterns show internal photogrammetric discrepancies. First, artefacts in the form of bands with sharp elevation change are explicitly visible in a NW-SE direction (e.g. Figure 9). Second, a series of parallel bands striking NE-SW show a sort of undulated pattern, (e.g. Figure 10). Third, distributed patches of strong elevation difference, both negative and positive, speckle steep slopes or river beds (e.g. Figure 9).

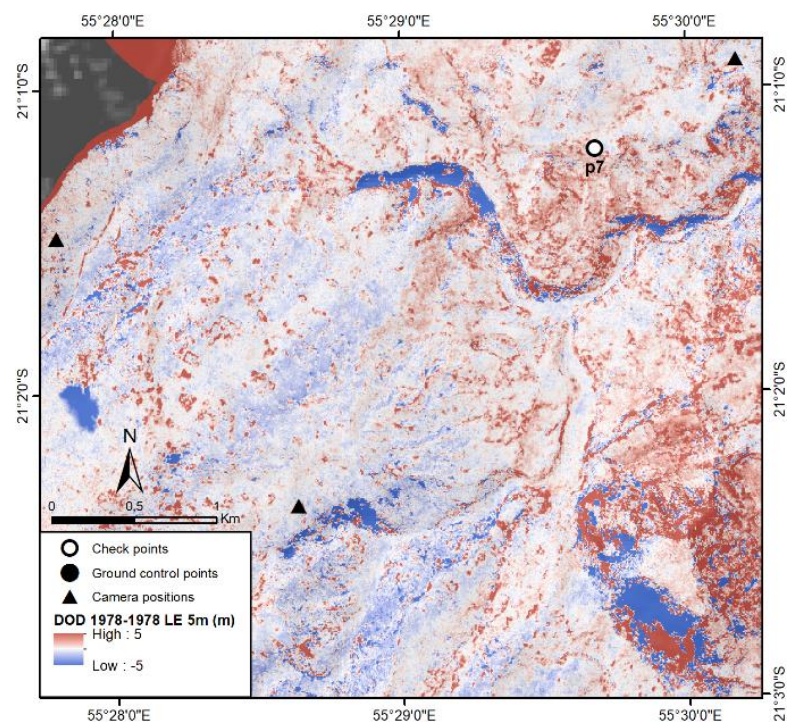

Figure 9. Difference of DEM at $5 \mathrm{~m}$ resolution (1978 all cameras1978 low elevation flight). $50 \mathrm{~m}$ elevation contours are in grey.

Elevation artefacts stand out. (i) two flight line edges appear in a NW-SE direction about $1.1 \mathrm{~km}$ apart from one another; (ii) stripes striking SW to NE show oscillations of elevation at $200-500 \mathrm{~m}$ wavelength.

In a perfect world, 1978 DSM elevation differences should have been next to zero. The high frequency "speckle-like" noise is clearly due to local elevation artefacts. Large zones of completely negative/positive elevation differences mark unresolved elevation for one of the DSM. They arise in areas of deep shadow or very steep slopes where stereoscopy is poor and image matching challenged by the lack of image texture. The stripy undulated pattern is a surprise to us. This looks like an interference pattern. We speculate that it results from photo scanning artefacts. Third, the most striking elevation jump marked along a NW-SE corridor (Figure 9) matches the sidelap corridor between two flight lines. This is the signature of imperfect orientation. We note that Riquelme et al.'s (2019) elevation comparisons also exhibit these artefacts from single flight coverages. This error could, in principle, have been mitigated with additional reference points in the side lap corridor. This emphasizes the importance of reference point distribution 
respective to photo positions (e.g. Kraus, 1993). If present, we note that this artefact is more conspicuous for all photos DSM, probably a benefit of better stereoscopy.

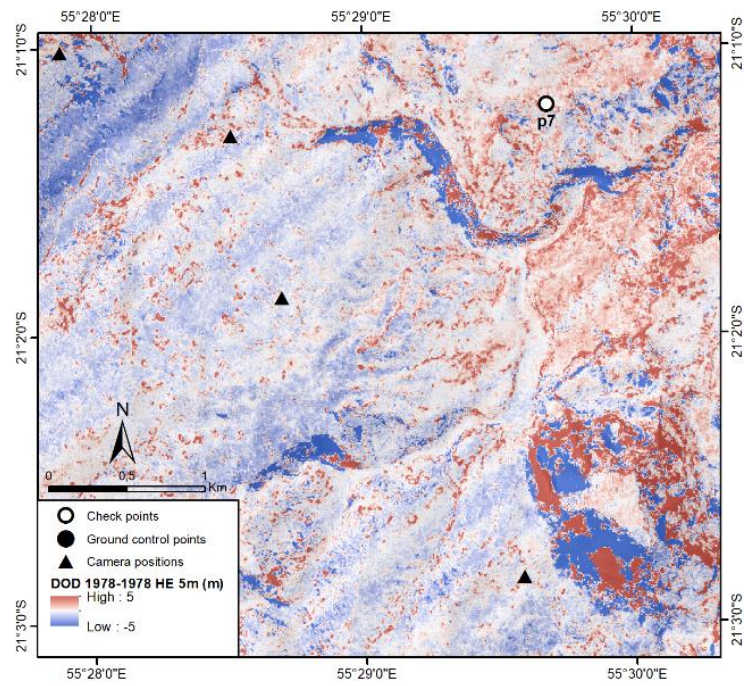

Figure 10. Difference of DEM at $5 \mathrm{~m}$ resolution (1978 all cameras DSM - 1978 high elevation DSM). Flight line artefact appear again in a NW-SE direction. Elevation oscillation stripes striking SW-NE also occur (see Figure 9) at a wavelength of $200-500 \mathrm{~m}$.

\section{AN IMAGE OF 37 YEARS OF MASS WASTING}

Beyond photogrammetric technicalities, there are several reasons why this work holds significant potential. The image revealed by Figure 6 is a complete map, to the limit of detection, of 37 years of cumulated mass wasting history in the vicinity of Grand-Ilet. Completeness was a notion difficult to argue when landslide maps were produced with stereoscopic interpretation of stereopairs. Here the coverage is total.

Going into further geomorphological considerations would be beyond the scope of this contribution. Before that, difference maps should be segmented into significant landslide objects, leading to individual volumetric estimates. Segmentation will make use of James et al.'s (2017) tie points covariance estimates to create photogrammetrically-aware and spatially variable detection thresholds. Once done, substantial work will be required for classifying the geomorphological phenomenon causing the elevation change, some signature may well be numerically significant, but spurious. Only then will a global sediment volume estimate make scientific sense.

To come back on the temporal perspective opened by this work, one should note that the most ancient dense native topographic data sets available for the whole of La Réunion island is the 2011 lidar coverage at 5-m-resolution. Prior to that, elevation information came from topographic contour maps and interpolated "coarse" (e.g. 10-50 m) Digital Elevation Models. With SFM now affording the -nearly- effortless extraction of sub-meter dense surface models of the past, mass wasting hazards assessment gains hindsight, a hindsight potentially going back seventy years, until 1949. The practical consequence lies in risk mitigation planning. To date, regulatory mapping is implemented on expert-say, relying on just a decade-long precise topographic data, and patchy qualitative accounts compilation for the longer term. Now, SFM combined with historical aerial photographs opens brand new avenues to protect goods and save lives.

\section{DISCUSSION AND CONCLUDING REMARKS}

In this contribution, we spent time exploring the effects of SFM processing on digital landscape reconstruction. Early papers evoking this topic (e.g. Gomez et al. 2015, Riquelme et al. 2019), marvelled at the possibility of doing so. They spent less time on deeper photogrammetric or SFM processing pipeline considerations.

Here, we show that image preparation is a significant source of improvement in the image geometry (image origin and image to camera axes alignments). An automatic Python code is used to produce images in a reproducible, unique and stable reference frame. Results show that raw scanned images may happen to produce 3D results with SFM by chance, but individual image origin shifts and rotations are bound to dramatically alter principal point estimates and the entire photogrammetric chain. Translation of origin, rotation and cropping are necessary.

With the level of residuals observed with reference points collected on IGN's national mapping portal, exterior orientation achieved accuracies better than $10 \mathrm{~m}$, with optimized pinpointing residuals at sub-pixel level. Resulting surface models are precise within $3.34 \mathrm{~m}$. This is a very encouraging result for producing Digital Surface Models from archive air photographs and go back in time to build landscape evolution chronicles. Epoch-to-epoch registration quality will be a quantification limit to compare data sets. We suggest here to align simultaneously several epochs in the same Metashape "chunk", albeit with different camera models if required (e.g. Cook and Dietze, 2019). In this way, common tie point matched automatically across epochs will take care of the relative registration. Based on several thousands common tie points, final adjustment ought to be far better than any manual registration point collection, or even posterior point cloud Iterative Closest Point registration.

We show that camera calibration parametrization only required 4 parameters (with $\mathrm{f}, \mathrm{cx}, \mathrm{cy}, \mathrm{K}_{1}$ ) to achieve optimal results without doming effect. Higher degree of parametrization (adding $\mathrm{K}_{2-3}$, and $\mathrm{P}_{1-2}$ ) was not necessary with this data set as parameters were correlated among them and reference point residuals were not improved by increasing the number of parameters.

Single flight camera networks are indeed geometrically weaker and more sensitive to alignment defects than multiple flights acquired over the course of a few days. The opportunistic redundant stereoscopic effect used in this study is most beneficial in controlling side lap adjustment and limiting flight line edges artefacts. In numerical terms, however, the benefits of increased stereoscopic coverage was not visible from estimated camera parameters or reference point adjustment quality figures.

Finally, comparison with recent Digital Terrain Model revealed a never-seen-before 37 years of intense mass wasting history in Cirque de Salazie. This image freezes the combined geomorphic signature of 13 cyclones intervened in the interval. Such data is a critical piece of information for the improvement of risk mitigation maps. It brings the hindsight of decades history. Our work, although mostly technical will have significant consequences for the risk management community.

\section{ACKNOWLEDGEMENTS}

Thanks to IGN for data distribution through remonterletemps.ign.fr and geoportail.gouv.fr. A. Eltner provided relevant and helpful comments. This study is part of RenovRisk-Erosion project funded by Reunion Island's Regional 
Council, European Union (FEDER), French Government and BRGM. In memory of J.-M Dewez who passed away when finalizing this work.

\section{REFERENCES}

Aguilar, M.A., Aguilar, F.J., Fernández, I., Mills, J.P., 2013. Accuracy Assessment of Commercial Self-Calibrating Bundle Adjustment Routines Applied to Archival Aerial Photography. Photogramm. Rec. 28, 96-114. https://doi.org/10.1111/j.14779730.2012.00704.x

Bakker, M., Lane, S.N., 2016. Archival photogrammetric analysis of river-floodplain systems using Structure from Motion (SFM) methods. Earth Surf. Process. Landforms 42, 1274-1286. https://doi.org/10.1002/esp.4085

Barrand, N.E., Murray, T., James, T.D., Barr, S.L., Mills, J.P., 2009. Optimizing photogrammetric DEMs for glacier volume change assessment using laser-scanning derived ground-control points. J. Glaciol. 55, 106-116. https://doi.org/10.3189/002214309788609001

Belle, P., Aunay, B., Bernardie, S., Grandjean, G., Ladouche, B., Mazué, R., Join, J.L., 2014. The application of an innovative inverse model for understanding and predicting landslide movements (Salazie cirque landslides, Reunion Island). Landslides 11, 343-355. https://doi.org/10.1007/s10346-0130393-5

Brown, D.C., 1971. Close- range camera calibration. Photogramm Eng.

Cook, K.L., 2017. An evaluation of the effectiveness of low-cost UAVs and structure from motion for geomorphic change detection. Geomorphology 278, 195-208. https://doi.org/10.1016/j.geomorph.2016.11.009

Cook, K.L., Dietze, M., 2019. Short Communication: A simple workflow for robust low-cost UAV-derived change detection without ground control points. Earth Surf. Dyn. Discuss. 1-15. https://doi.org/10.5194/esurf-2019-27

Dewez, T.J.B., Leroux, J., Morelli, S., 2016. Cliff collapse hazard from repeated multicopter uav acquisitions: Return on experience. Int. Arch. Photogramm. Remote Sens. Spat. Inf. Sci. ISPRS Arch. 41, 805-811. https://doi.org/10.5194/isprsarchives-XLI-B5-805-2016

Eltner, A., Kaiser, A., Castillo, C., Rock, G., Neugirg, F., Abellán, A., 2016. Image-based surface reconstruction in geomorphometry-merits, limits and developments. Earth Surf. Dyn. 4, 359-389. https://doi.org/10.5194/esurf-4-359-2016

Eltner, A., Schneider, D., 2015. Analysis of Different Methods for 3D Reconstruction of Natural Surfaces from Parallel-Axes UAV Images. Photogramm. Rec. 30, 279-299. https://doi.org/10.1111/phor.12115

Fonstad, M.A., Dietrich, J.T., Courville, B.C., Jensen, J.L., Carbonneau, P.E., 2013. Topographic structure from motion: A new development in photogrammetric measurement. Earth Surf. Process. Landforms 38, 421-430. https://doi.org/10.1002/esp.3366

Gomez, C., Hayakawa, Y., Obanawa, H., 2015. A study of Japanese landscapes using structure from motion derived DSMs and DEMs based on historical aerial photographs: New opportunities for vegetation monitoring and diachronic geomorphology.

https://doi.org/10.1016/j.geomorph.2015.02.021
Höhle, J., Höhle, M., 2009. Accuracy assessment of digital elevation models by means of robust statistical methods. ISPRS J. Photogramm. Remote Sens. 64, 398-406. https://doi.org/10.1016/j.isprsjprs.2009.02.003

James, M.R., Robson, S., 2014a. Sequential digital elevation models of active lava flows from ground-based stereo time-lapse imagery. ISPRS J. Photogramm. Remote Sens. 97, 160-170. https://doi.org/10.1016/j.isprsjprs.2014.08.011

James, M.R., Robson, S., 2014b. Mitigating systematic error in topographic models derived from UAV and ground-based image networks. Earth Surf. Process. Landforms 39, 1413-1420. https://doi.org/10.1002/esp.3609

James, M.R., Robson, S., 2012. Straightforward reconstruction of 3D surfaces and topography with a camera: Accuracy and geoscience application. J. Geophys. Res. Earth Surf. 117, 1-17. https://doi.org/10.1029/2011JF002289

James, M.R., Robson, S., Smith, M.W., 2017. 3-D uncertaintybased topographic change detection with structure-from-motion photogrammetry: precision maps for ground control and directly georeferenced surveys. Earth Surf. Process. Landforms 42, 1769-1788. https://doi.org/10.1002/esp.4125

Kraus, K., 1993. Photogrammetry, Volume I. Ferd, Volum I. ed. Dummlers Verlag, Bonn, Germany.

Ma, Y., Kosecka, J., Soatto, S., Sastry, S., 2004. An invitation to 3-d vision: from images to geometric models. https://doi.org/10.1007/978-0-387-21779-6

Micheletti, N., Lane, S.N., Chandler, J.H., 2015. Application of archival aerial photogrammetry to quantify climate forcing of alpine landscapes. Photogramm. Rec. 30, 143-165. https://doi.org/10.1111/phor.12099

Pinchinot, H., 1984. Etude geologique des formations superficielles et du proche substratum à Grand Ilet ( cirque de Salazie - La Réunion ) application a la cartographie du risque de mouvements de versants To cite this version: HAL Id: tel00679334. Université Scientifique et Médicale de Grenoble.

Riquelme, A., Del Soldato, M., Tomás, R., Cano, M., Jordá Bordehore, L., Moretti, S., 2019. Digital landform reconstruction using old and recent open access digital aerial photos. Geomorphology 329, 206-223. https://doi.org/10.1016/j.geomorph.2019.01.003

Smith, M.W., Vericat, D., 2015. From experimental plots to experimental landscapes: Topography, erosion and deposition in sub-humid badlands from Structure-from-Motion photogrammetry. Earth Surf. Process. Landforms 40, 16561671. https://doi.org/10.1002/esp.3747

Wenzel, K., Rothermel, M., Fritsch, D., Haala, N., 2013. Image Acquisition and Model Selection for Multi-View Stereo. ISPRS - Int. Arch. Photogramm. Remote Sens. Spat. Inf. Sci. XL-5/W1, 251-258. https://doi.org/10.5194/isprsarchives-xl-5-w1-2512013

Westoby, M.J., Brasington, J., Glasser, N.F., Hambrey, M.J., Reynolds, J.M., 2012. "Structure-from-Motion" photogrammetry: A low-cost, effective tool for geoscience applications. Geomorphology 179, 300-314. https://doi.org/10.1016/j.geomorph.2012.08.021 